\title{
Depression, smoking abstinence and HPA function in women smokers
}

\author{
Ovide F. Pomerleau*, Cynthia S. Pomerleau, Sandy M. Snedecor, Stefanie Gaulrapp, \\ Rebecca Namenek Brouwer ${ }^{\dagger}$ and Oliver G. Cameron \\ Department of Psychiatry, Nicotine Research Laboratory, University of Michigan, Ann Arbor, MI 48105, USA
}

\begin{abstract}
To determine whether smokers with a history of depression are differentially susceptible to smoking withdrawal, depressed mood induction and/or hypothalamic-pituitary-adrenal (HPA) axis dysregulation during smoking abstinence, 24 women smokers with and without such a history were studied. During one 5-day interval, participants smoked ad libitum; during a second they abstained. On day 4, the participants were exposed to the Velten mood induction procedure (VMIP). Participants were then instructed to take $1 \mathrm{mg}$ dexamethasone at $11 \mathrm{pm}$. At $4 \mathrm{pm}$ on day 5, blood samples were withdrawn to determine the cortisol and ACTH response. Despite lower baseline cotinine levels, history-positive participants displayed more pronounced overall withdrawal distress than did history-negative participants, regardless of condition. The VMIP increased depression as well as negative responses on other profile of mood states subscales. Despite many overall group differences, no significant main effects for smoking condition nor interaction effects emerged. All participants evinced cortisol suppression in response to dexamethasone during both conditions, but the degree of suppression did not differ as a function of either abstinence or depression history. In history-positive smokers, however, ACTH levels trended toward overall elevation and showed almost no suppression during abstinence; thus exacerbation of HPA dysregulation in historypositive smokers during smoking abstinence cannot be ruled out. Copyright (C) 2004 John Wiley \& Sons, Ltd.
\end{abstract}

KEY WORDS - smoking; nicotine withdrawal; depression; dexamethasone suppression test (DST); cortisol; adrenocorticotropic hormone $(\mathrm{ACTH})$

\section{INTRODUCTION}

Considerable evidence has been presented that smoking is overrepresented in individuals with diagnosable or subclinical depression, or even with a history of depression (Anda et al., 1990; Covey et al., 1990; Glassman, 1993). And although the evidence is not unequivocal (e.g. Ginsberg et al., 1995; Hall et al., 1994; Hitsman et al., 2001), a number of studies have shown that these smokers have greater difficulty in quitting (e.g. Glassman et al., 1988; Glassman,

\footnotetext{
* Correspondence to: Dr O. F. Pomerleau, Nicotine Research Laboratory, 2025 Traverwood Drive, Ste B, Ann Arbor, MI 48105, USA. Tel: 734-998-6435. Fax: 734-998-6443.

E-mail: ofpom@umich.edu

${ }^{\dagger}$ Cancer Prevention, Detection and Control Research Program, Comprehensive Cancer Center, Duke University Medical Center, Durham, NC 27710, USA.

Contract/grant sponsor: National Cancer Institute; contract/grant number: CA42730.
}

1993; Balabanis et al., 2001). To the extent that they are successful, their quit attempts may be complicated by the emergence of greater and more persistent withdrawal symptomatology, particularly depressed mood (e.g. Borrelli et al., 1996; Covey et al., 1990; Hall et al., 1991; Kinnunen et al., 1996; Niaura et al., 1999; Pomerleau et al., 2001; Glassman et al., 2001) - although a higher likelihood of episodes of depression in such smokers even when they are not attempting to quit has also been noted (Tsoh et al., 2000). An additional consideration is that depression is more common in women than in men, with women accounting for $61.2 \%$ of the total in lifetime prevalence of depressive symptoms and $71.2 \%$ of major depressive disorder (MDD)/dysthymia (Johnson et al., 1992; Weissman et al., 1991). Moreover, although the association of smoking with depression has been shown to be significant for both men and women, data from the National Health and Nutrition Epidemiological Survey (Anda et al., 1990), the St. Louis ECA study (Glassman et al., 1990) and the San Francisco survey 
(Perez-Stable et al., 1990) suggest that this relationship may be more pronounced in women than in men.

People with a history of MDD are more susceptible to HPA-axis dysregulation (Carroll et al., 1981; Young et al., 1991,) and clinical observations by Glassman (1993) suggest that chronic nicotine exposure may help to maintain affective equilibrium and 'normalize' HPA-axis function in certain people. Thus, when smokers with a history of MDD stop smoking, in addition to manifestations of clinical depression or severe dysphoria, there may be a rebound effect on the HPA-axis resulting in a loss of feedback inhibition of cortisol. Resumption of smoking has been found to terminate depression/dysphoria (Fagerström et al., 1991) - within several hours in some cases (Glassman, 1993). While much uncertainty remains about biobehavioral mechanisms that might link smoking and depression (see Carmody, 1989), nicotine is known to have important effects on central acetylcholine and catecholamines (Pomerleau and Pomerleau, 1984), both of which have been shown to play a role in the etiology of depression (Siever, 1987; Janowsky and Risch, 1987); moreover, nicotine is known to affect brain regions that influence mood and well-being (Carmody, 1989; Pomerleau and Rosecrans, 1989). It is plausible, therefore, that the same genetic variations in brain neurotransmitter systems that influence the probability of major depression also increase the probability of smoking by enhancing the degree to which nicotine provides reinforcement via affective normalization (Kendler et al., 1993). Further, in people whose psychological and/or physiological adaptability is compromised, nicotine may serve to maintain homeostasis in critical systems such as the HPA-axis; for such people, nicotine use may constitute a coping strategy for meeting the challenges of daily living (see Pomerleau and Pomerleau, 1984).

In order to determine whether the reinforcing value of nicotine self-administration is enhanced in depressed individuals by the drug's affect-normalizing properties and its ability to protect against the hypothalamic-pituitary-adrenal (HPA) axis dysregulation that often characterizes episodes of depression-as indicated by elevated cortisol and ACTH levels following dexamethasone administration-a laboratory test was conducted of women smokers with and without a history of depression. We also wished to determine susceptibility to induction of depressed mood in history-positive and history-negative women smokers. Participants were studied during ad libitum smoking and over the first few days of abstinence from smoking, the interval during which, despite consider- able individual variability (Piasecki et al., 2000), craving and cognitive/affective withdrawal symptomatology typically peak (Ward et al., 2001).

Our expectations were as follows: (1) Over the course of 3 days of smoking abstinence, history-positive (HX+MDD) smokers would experience withdrawal symptomatology of greater magnitude and duration than history-negative (HX-MDD) smokers; (2) After 3 days of abstinence from smoking, historypositive smokers would be more susceptible to induction of dysphoria than would history-negative smokers; (3) After 4-5 days of abstinence from smoking, history-positive smokers would show HPA dysregulation as indicated by post-DST cortisol and ACTH levels, whereas history-negative smokers would show little or no evidence of HPA dysregulation.

\section{METHODS}

\section{Participants}

Twenty-four female smokers, 12 with and 12 without a history of depression, were recruited from the general community through local newspaper advertisements and posters. Participants were required to meet the following criteria: between 20 and 55 years of age; premenopausal; in good health; weight $\geq 100$ pounds; score $\geq 3$ on the Fagerström test of nicotine dependence (FTND; Fagerström et al., 1991; Heatherton et al., 1991; Pomerleau et al., 1994); smoking at least 10 cigarettes per day; and smoking at the current rate for at least 1 year. Candidates were excluded for the following: current use of CNS or cardiovascular-acting drugs; current use of antidepressant or antipsychotic medication; cardiac, vascular, pulmonary or gastric disease; hypertension; history of throat irritation or severe sinus infection; allergy to nicotine; history of seizures; history of abuse of drug or alcohol within 1 year prior to the study; history of anorexia nervosa; $\geq 15 \%$ below normal weight; regular use of other tobacco products such as cigars or pipes; and current depression (episode within past 6 months). Candidates were also excluded if they were currently pregnant or breastfeeding, had been pregnant or breastfeeding within the past 3 months, or were at risk of becoming pregnant.

A total of 643 interested people were telephonescreened, 147 of whom met preliminary qualifications and were invited to come into the laboratory for a screening interview. Of these, 35 opted not to participate further, 24 failed to show up for an appointment, $41 \mathrm{kept}$ the appointment but were disqualified due to 
smoking criteria or medical reasons, and the remaining 47 candidates qualified for participation in the study. Of these, eight lost interest and/or did not show up for scheduled laboratory sessions, seven could not be re-contacted after the initial interview, two decided that they did not have enough time to participate, two smoked during the abstinence phase and had to be disqualified, two were dropped due to problems maintaining adequate blood flow during laboratory sessions, and two could not be enrolled because they failed to qualify for unfilled cells; ultimately, 24 participants completed the protocol.

\section{Procedure}

The protocol for this study was approved by the Institutional Review Board of the University of Michigan Medical School. All participants meeting preliminary qualifications from the telephone screen were scheduled for a screening interview and sent a packet that included the Milcom Health History Questionnaire and baseline questionnaires which included the following: general history (to assess demographics); smoking history; substance intake history; Center for Epidemiological Studies-Depression (CES-D; Radloff, 1977); and the Fagerstrom test of nicotine dependence (FTND; Fagerstrom et al., 1991; Heatherton et al., 1991; Pomerleau et al., 1994).

At the screening interview, the study was explained and informed consent obtained. The Milcom was reviewed to determine medical exclusion, height, weight and blood pressure were measured, a pregnancy test was administered, and a $5 \mathrm{ml}$ blood sample was withdrawn for baseline cotinine concentrations. The structured clinical interview for DSM-IV (SCID; First et al., 1994) was conducted by a trained interviewer to determine history of major depressive disorder, mania, hypomania, dysthymia and anorexia nervosa. Acceptance into the HX+MDD or HX-MDD groups was made on the basis of this interview. To be categorized as $\mathrm{HX}+\mathrm{MDD}$, participants were required to have experienced at least one episode of MDD with five or more symptoms within the past 10 years. To rule out currently depressed individuals, they were required not to have had an episode of MDD or met criteria for dysthymia within the past 6 months. To qualify as HX-MDD, smokers could not have a history of any depression diagnosis.

The study consisted of two 5-day intervals separated by approximately a month and timed to coincide with the mid- to late-follicular phase (operationally, between the end of menses and day 11 of the menstrual cycle) - the time when affective, physiological and hormonal responses are known to be 'low' or neutral (Schechter et al., 1989). During one 5-day interval the participant smoked ad libitum; during the other 5day interval, she abstained from smoking; conditions were presented in counterbalanced order. CO levels $(\mathrm{CO} \leq 10)$ were measured on all 5 days, and a urine sample was obtained on day 3 to rule out pregnancy. On days 4 and 5 of the ad libitum smoking interval, to maintain a steady state of nicotine during laboratory testing, the participant was provided with two $15 \mathrm{mg}$ Nicotrol $\AA$ nicotine transdermal patches to be worn from $1100 \mathrm{~h}$ through to the end of the laboratory session. On days 3-5 of the abstinence interval, the participant also provided a urine sample for testing with a NicCheck ${ }^{\mathrm{TM}}$ test strip for nicotine and its metabolites for additional verification of abstinence.

For the first 3 days of each interval, the participant came into the laboratory to receive/drop off her daily diary questionnaire. This instrument was completed at bedtime and consisted of a 24-h retrospective assessment of smoking craving and withdrawal symptomatology as specified in DSM-IV (APA, 1994) depressed mood, insomnia, irritability, anxiety, difficulty concentrating, restlessness, increased appetitemeasured on a bipolar scale of -5 to +5 with values less than zero recoded as zero (Pomerleau et al., 2001).

On day 4 of each phase, the participant reported for a laboratory session that began at $1500 \mathrm{~h}$. The participant was seated in a recliner on one side of the oneway mirror; an intravenous catheter, attached to infusion-exfusion tubing to allow unobtrusive withdrawal in an adjacent room, was inserted $60 \mathrm{~min}$ prior to blood sampling to allow time for cortisol levels to stabilize. Samples for cortisol and nicotine/cotinine were withdrawn at $1600 \mathrm{~h}$; samples for ACTH were withdrawn four times over a 20 min interval and pooled. The line was heparinized between draws to prevent clotting. Samples were collected in standard EDTA vacutainer tubes which were kept on ice during the session, centrifuged at $4^{\circ} \mathrm{C}$, with the plasma stored at $-80^{\circ} \mathrm{C}$ until assayed. Plasma cortisol levels were measured by RIA, using Diagnostic Product Corporation's cortisol CORT-A-COUNT kit. Plasma ACTH levels were determined using a Nichols Institute Allegro HS-ACTH immunoassay kit. Baseline cotinine was measured using high performance liquid chromatography (HPLC; Hariharan et al., 1988).

The session lasted approximately $3 \mathrm{~h}$, during which a modified version the Velten mood induction procedure (VMIP; Velten, 1968) was used as a provocative assessment of susceptibility to dysphoria. The standard VMIP induces affect by having participants read 
a series of 50 emotional statements, with encouragement to actualize the feelings suggested by each statement. In the modified version, a pre-recorded tape was broadcast via speakers placed in the laboratory, consisting of $15 \mathrm{~min}$ of neutral statements followed by 15 min of dysphoric-mood statements. The Profile of Mood States (POMS; McNair et al., 1971) was administered once before the neutral statements, once between the two segments, and once after the depression statements, with the following instructions: 'Please read EVERY word carefully. Then fill in ONE space under the answer which best describes how you feel RIGHT NOW. Mark the answer which is closest to how you feel RIGHT NOW using the following numbers....'

Following the session, the participant was given a $1 \mathrm{mg}$ dexamethasone tablet with instructions to take it at $2300 \mathrm{~h}$-the standard protocol for the dexamethasone suppression test (DST; Carroll et al., 1981). The DST, which involves monitoring the cortisol response to the administration of a small amount of the exogenous glucocorticoid, dexamethasone, was used to examine systematically the relationship between abstinence-induced dysphoria and dysregulation of inhibitory feedback mechanism in the hypothalamic-pituitary-adrenal (HPA) axis. Depressed individuals are less likely to show the normal response of cortisol suppression to levels below $1 \mu \mathrm{g} / \mathrm{dl}$ (Baumgartner et al., 1985) and also tend to show enhanced variability in the range of cortisol response. ACTH release was also measured in order to provide additional information about inhibitory feedback mechanisms.

On day 5 of each phase, the participant reported to the laboratory at $1500 \mathrm{~h}$ and a catheter was inserted as described above. After $60 \mathrm{~min}$, a single sample for post-dexamethasone cortisol was withdrawn, and four samples for ACTH were taken over a 20 min interval for subsequent pooling.

\section{Data analysis}

Baseline differences between diagnostic groups were compared using independent $t$-tests and ChiSquare tests as appropriate. Because of marginally significant differences in baseline cotinine levels, baseline concentrations were covaried in all subsequent analyses. Tests of withdrawal symptoms over the first 3 days were conducted using SAS PROC MIXED. Because preliminary testing revealed no higher-order interaction for any variable, diagnostic group, smoking condition, day and all first-order interactions were included in the model. Session POMS data were ana- lysed including diagnostic group, smoking condition, time (pre- and post-mood induction), and all firstorder interactions, using baseline (pre-neutral statements) as an additional covariate. Cortisol and ACTH values were log-transformed for the purpose of statistical testing. Because of the potential effect of age on DST results (Akil et al., 1993), and because of substantial though nonsignificant group differences in age, this variable was also included as a covariate. Because of the possible impact of oral contraceptive use on cortisol and ACTH levels (Kirshbaum et al., 1999), two oral contraceptive users matched for group and contraceptive type (Triphasic) were included in analyses of these variables; a third oral contraceptive user, in the HX+MDD group, could not be matched and was excluded. Data were analysed using SAS PROC MIXED, with diagnostic group, smoking condition, dexamethasone (pre vs post) and all first-order interactions included in the model.

\section{RESULTS}

\section{Baseline group differences}

Baseline characteristics for the two groups are shown in Table 1. CES-D scores for HX+MDD participants were higher than for HX-MDD. The only other significant difference was that cotinine levels were higher in HX-MDD participants.

In the HX+MDD group, four women had had a single episode of MDD, seven had had two episodes, and one had had three. The mean length of time since the most recent episode was $2.4 \pm 2.4$ years (range $0-8$ years).

Compared with participants who completed the protocol, the 23 individuals who enrolled but either did not start or did not complete the protocol were significantly older (completers: $31.0 \pm 9.5$ years; noncompleters: $39.1 \pm 12.4$ years; $t=-2.53, p<0.05$ ) and more nicotine dependent as measured by the

Table 1. Participant characteristics (mean $\pm \mathrm{SEM}$ or percent; $t$-test or ChiSquare)

\begin{tabular}{lccc}
\hline & $\begin{array}{c}\text { HX }+ \text { MDD } \\
(n=12)\end{array}$ & $\begin{array}{c}\text { HX }- \text { MDD } \\
(n=12)\end{array}$ & $p$-value \\
\hline Age (years) & $34.0 \pm 2.7$ & $28.0 \pm 2.5$ & $\mathrm{NS}$ \\
Race $(\%$ white) & $83 \%$ & $83 \%$ & $\mathrm{NS}$ \\
BMI $\left(\mathrm{kg} / \mathrm{m}^{2}\right)$ & $25.2 \pm 1.1$ & $27.2 \pm 1.6$ & $\mathrm{NS}$ \\
Smoking rate & $17.8 \pm 2.3$ & $20.0 \pm 1.5$ & $\mathrm{NS}$ \\
(cigarettes/day) & & & \\
Cotinine (ng/ml) & $186.7 \pm 27.2$ & $324.3 \pm 61.0$ & $<0.10$ \\
FTND (range 0-10) & $4.2 \pm 0.5$ & $5.2 \pm 0.5$ & $\mathrm{NS}$ \\
CES-D (range 0-60) & $19.7 \pm 3.5$ & $6.6 \pm 1.8$ & $<0.05$ \\
\hline
\end{tabular}


FTND (completers: $4.7 \pm 1.8$; noncompleters: $6.0 \pm 2.2 ; t=-2.26, p<0.05)$. They did not differ significantly with respect to race distribution or cigarettes/day.

\section{Craving and withdrawal effects over the first three days}

Craving and withdrawal symptomatology over the 3day pre-session period are shown in Figure 1. For craving, a significant main effect emerged for condition (smoking vs abstinence; $F[1,20]=21.94$, $p<0.0001$ ), with a marginal interaction of condition with diagnostic group $(\mathrm{F}[1,20]=3.78, p<0.10)$, such that craving during abstinence was most strongly elevated in HX-MDD. Significant or marginal main effects were detected for both diagnostic group and condition for irritability (diagnostic group: $F[1,22]$ $=6.33, p<0.05$; condition: $\mathrm{F}[1,20]=14.75, p<$ 0.01 ), anxiety (diagnostic group: $\mathrm{F}[1,22]=3.45$, $p<0.10$; condition: $\mathrm{F}[1,20]=19.38, p<0.001$ ), difficulty concentrating (diagnostic group: $\mathrm{F}[1,22]=3.26$, $p<0.10$; condition: $\mathrm{F}[1,20]=10.43, p<0.01)$, and restlessness (diagnostic group: $\mathrm{F}[1,22]=5.35$, $p<0.05$; condition: $\mathrm{F}[1,20]=19.08, p<0.001)$. Significant or marginal main effects for diagnostic group only were detected for depression $(F[1,22]=13.76$, $p<0.01)$ and insomnia $(\mathrm{F}[1,22]=3.27, p<0.10)$. Significant main effects for condition only were detected for increased appetite $(F[1,20]=14.72$, $p<0.01)$. Except for a marginal diagnostic group by day interaction for insomnia $(\mathrm{F}[2,44]=2.74$, $p<0.10$ ), no interaction effects for withdrawal symptoms were observed.

\section{Response to mood induction}

Response to mood induction is shown in Figure 2. A time effect was observed for the POMS elated/ depressed subscale $(\mathrm{F}[1,22]=42.67, p<0.0001)$, indicating that the mood induction procedure was successful. Significant differences were also observed for diagnostic group $(\mathrm{F}[1,22]=9.05, p<0.01)$, with the HX+MDD group evincing a greater overall level of depression than the HX-MDD group. Regarding the remaining scales, significant time effects were also observed for composed/anxious $(\mathrm{F}[1,22]=4.72, p<$ $0.05)$, agreeable/hostile $(\mathrm{F}[1,22]=15.80, p<0.001)$, onfident/unsure $\quad(\mathrm{F}[1,22]=9.11, \quad p<0.01) \quad$ and energetic/tired $(F[1,22]=18.81, p<0.001)$. Significant group differences were detected for agreeable/ hostile $(\mathrm{F}[1,22]=7.28, \quad p<0.05)$, with a trend towards significant differences for energetic/tired
$(F[1,22]=2.97, p<0.10)$. No significant effects for smoking condition or for any interaction for elated/ depressed or any other POMS scale were observed.

\section{Cortisol and ACTH}

Data for $11 \mathrm{HX}-\mathrm{MDD}$ and $11 \mathrm{HX}+\mathrm{MDD}$ participants were available for analysis of cortisol; an additional participant had missing ACTH data, leaving 10 cases in the HX + MDD group available for ACTH analysis. The results for cortisol and ACTH are shown in Figure 3. Comparison of pre-session values showed no significant differences based on either smoking condition or depression status for either cortisol or ACTH (expressed as continuous variables). Comparisons of pre- vs post-dexamethasone administration showed highly significant postdexamethasone suppression of both cortisol $(\mathrm{F}[1,20]=370.83, p<0.0001)$ and ACTH $(\mathrm{F}[1,19]$ $=27.72, p<0.0001)$; no participant in either group exhibited DST nonsuppression during either the smoking or abstinence condition, using $5 \mu \mathrm{g} / \mathrm{dl}$ as the cutoff. For ACTH, but not cortisol, a trend towards significant between-groups differences was detected $(\mathrm{F}[1,18]=3.20, \quad p<0.10)$, with levels for the HX+MDD group exceeding those for the HX-MDD MDD group. No significant differences or trends were detected for either cortisol or ACTH for smoking condition (ad libitum smoking vs abstinence) or any interaction.

\section{DISCUSSION}

Baseline characteristics for HX+MDD and HX-MDD women smokers were similar in most respects. The HX+MDD participants exhibited higher CES-D scores than the HX-MDD participants but this was expected given that they were selected on the basis of diathesis for depression. Cotinine levels showed a trend towards being higher in the HX-MDD participants, which was unrelated to the selection criteria and seems adventitious; differences in cigarettes/day or degree of dependence were in a consistent direction but fell short of being a trend.

As expected, significant main effects for condition (smoking vs abstinence) were detected for craving and most withdrawal symptoms. More surprisingly, regardless of smoking condition, we found greater overall levels of affective smoking-withdrawal symptomatology (irritability, anxiety, difficulty concentrating, restlessness, depression and insomnia) in historypositive participants despite their lower baseline levels of nicotine intake. Withdrawal symptomatology 

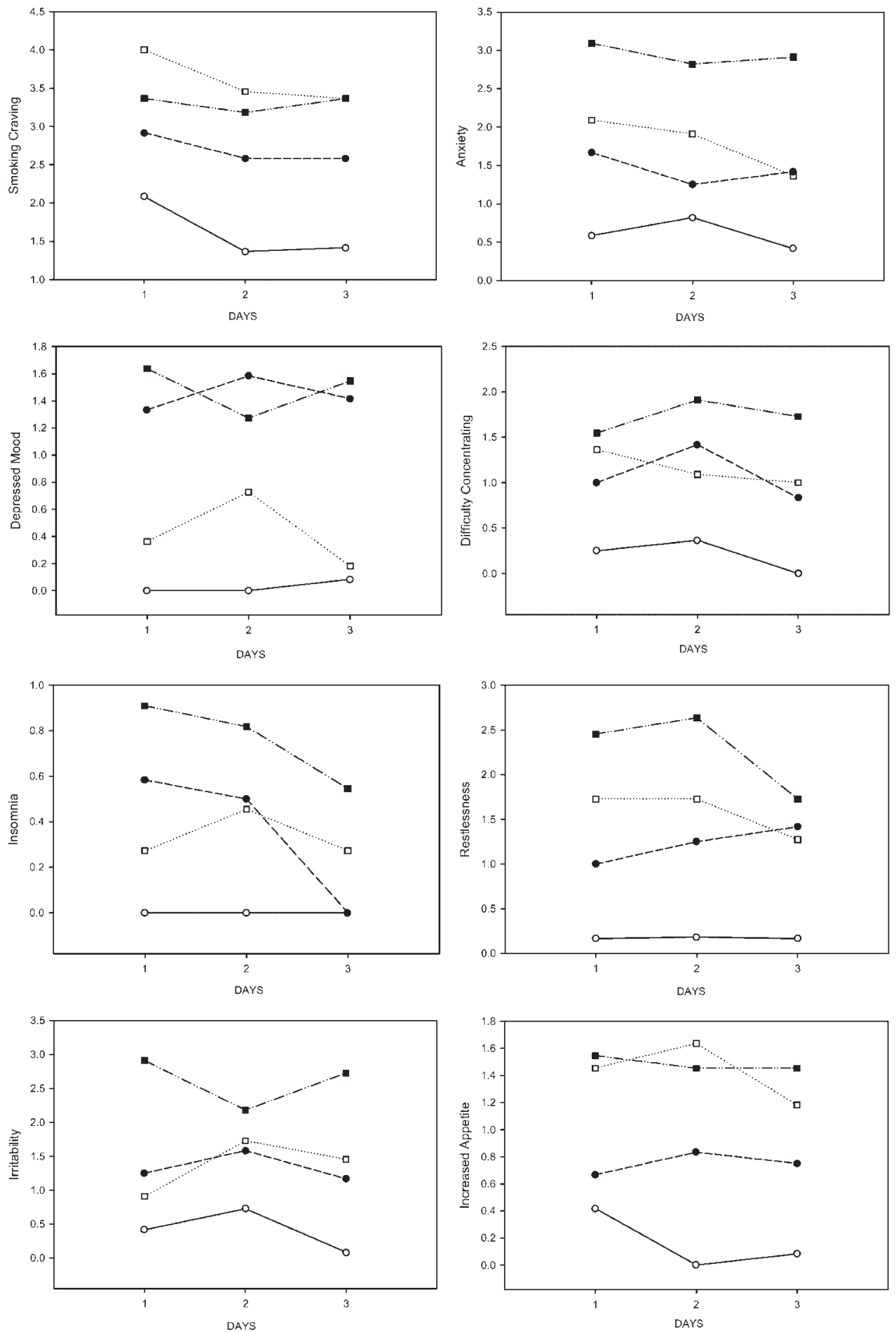

Figure 1. Craving and withdrawal symptoms over 3 days (means on a scale of 0-5). Open circles: HX-MDD, ad libitum smoking; open squares: HX-MDD, smoking abstinence; filled circles: HX+MDD, ad libitum smoking; filled squares: HX+MDD, smoking abstinence 

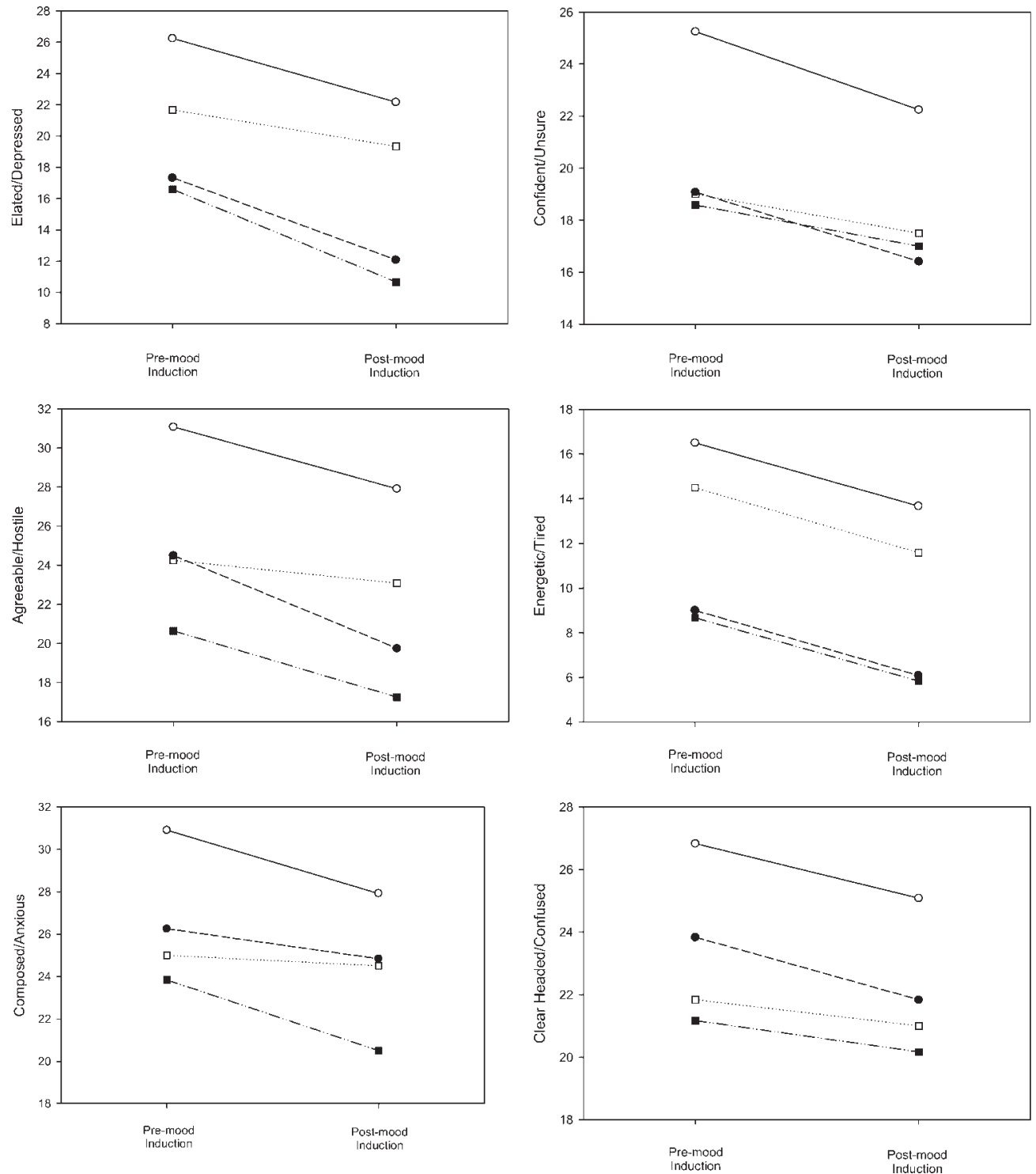

Figure 2. Mood ratings on POMS subscales (means on scales of 0-36) before and after the Velten mood induction procedure (day 4). Open circles: HX-MDD, ad libitum smoking; open squares: HX-MDD, smoking abstinence; filled circles: HX+MDD, ad libitum smoking; filled squares: $\mathrm{HX}+\mathrm{MDD}$, smoking abstinence

has been reliably demonstrated even in minimally deprived smokers, being lowest immediately after smoking and rising measurably within half an hour to an hour (Pomerleau et al., 1983), at which point dependent smokers typically light up another cigarette. Although no differential effects were observed based on several days' abstinence vs ad libitum smoking, our findings suggest that individuals with a diathesis for depression may experience more chronic distress from withdrawal symptomatology than their nondepressed counterparts. This circumstance may contribute to relapse, unsuccessful quitting, or failure 


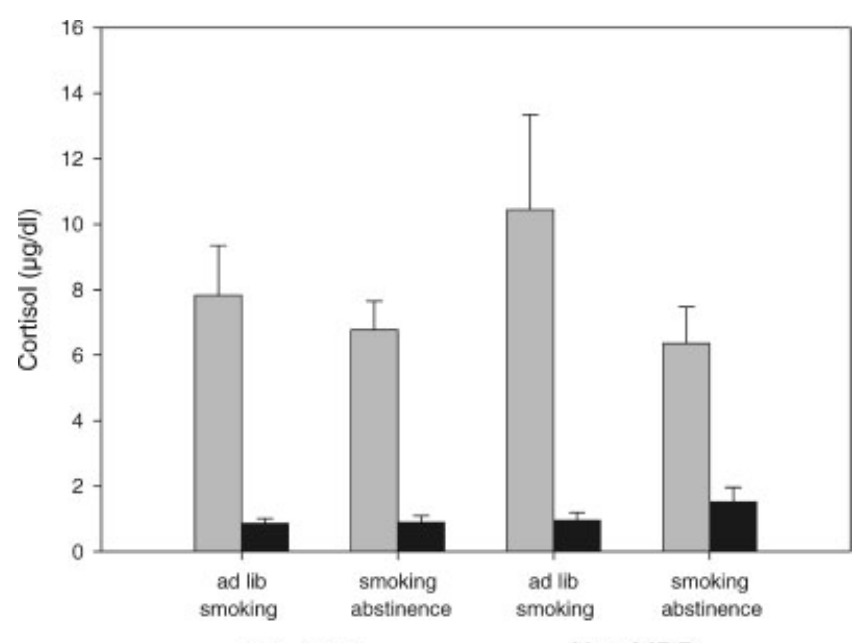

$\mathrm{Hx}-\mathrm{MDD}$

$\mathrm{Hx}+\mathrm{MDD}$

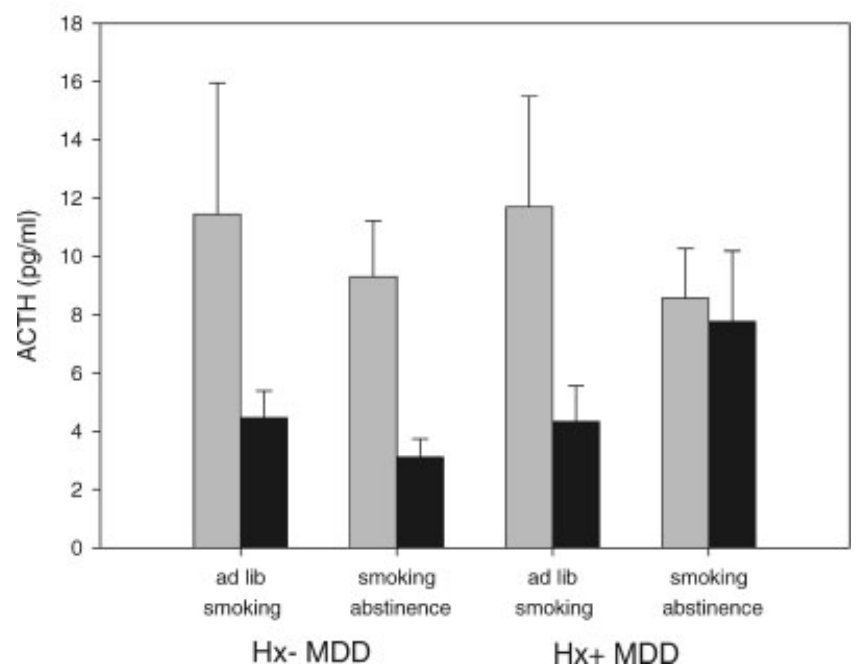

Figure 3. Pre- and post-DST levels of cortisol and ACTH (mean \pm SEM) (days 4 and 5). Shaded bars: pre-dexamethasone condition; dark bars: post-dexamethasone condition

even to initiate a quit attempt in smokers with a history of depression. By contrast, craving did not differ based on diagnostic group, and indeed, a marginal interaction of condition with diagnostic group emerged such that craving during abstinence was most strongly elevated in nondepressed smokers-probably reflecting their higher nicotine intake as indicated by baseline cotinine levels. This disjunction between craving and affective withdrawal symptoms lends further support to our contention that these patterns may represent distinct phenotypes for smoking (e.g. Pomerleau et al., 2000).

The modified Velten mood induction procedure was effective in increasing self-reported depressed mood, as measured by the POMS elated/depressed subscale in both history positive and history negative participants, and also increased negative responses on several other POMS subscales. A number of overall group differences were observed, suggesting that the HX+MDD group may have experienced greater depression than the HX-MDD group. No significant main effects for smoking condition nor any interaction effects emerged, however; little evidence was found for a differential effect of abstinence on susceptibility to acute mood induction by history of depression.

The results for HPA axis hormones are not as easily characterized. Regarding the effects of smoking 
abstinence on cortisol, conflicting evidence has been presented in the literature. In two previous studies involving the DST in smokers, for example, Hughes et al. (1988) found a significant increase in baseline (pre-dexamethasone) cortisol in nondepressed laboratory participants after 1-2 days' abstinence, whereas Frederick et al. (1998) found a significant decrease in baseline cortisol in quitting smokers at 2 and 4 weeks. In our study, in which confirmed abstinence was required and history of depression was included as a grouping factor, there were no changes in baseline cortisol or ACTH levels after 4 days' abstinence. Although our findings cannot be regarded as conclusive in light of discrepancies in the previously cited studies, they indicate that investigations using repeated measures over both short- and long-term abstinence will be needed to resolve the issue.

All participants in our study evinced cortisol suppression in response to dexamethasone (i.e. none escaped from suppression) during both smoking and abstinence conditions (using $5 \mu \mathrm{g} / \mathrm{dl}$ as the cutoff), and no differences were found in degree of suppression as a function of either abstinence or history of depression. An explanation proposed by Hughes et al. (1988) upon failure to observe elevated cortisol levels following dexamethasone administration during 1-2 days' smoking abstinence in nondepressed smokers is that, as with baseline cortisol, a longer period of abstinence may be needed to elicit HPA dysregulation. No changes were found in degree of suppression over time over 5 days' abstinence, however, and Frederick et al. (1998) found no interaction for time by percent suppression over 2 and 4 weeks after quitting, suggesting that time alone may not be the critical variable.

Another and perhaps more likely possibility is that a negative bias may have been introduced by the study design: Not only were persons with current diagnosis of major depression and/or taking an antidepressant excluded, but also participants were told they would be abstaining only for a time-limited interval rather than actually quitting smoking. These methodological considerations, though conservative, may have limited the ability of the present design to elicit a degree of depression/dysphoria sufficient to precipitate escape from dexamethasone suppression. We note, moreover, that only $40 \%-$ $50 \%$ of patients with current diagnosis of MDD typically exhibit escape from dexamethasone suppression when tested (Ribeiro et al., 1993), suggesting that the DST in its present form may not be sensitive enough to detect more subtle degrees of HPA dysre- gulation accompanying moderate depression/dysphoria induced by smoking abstinence. Providing some support for this speculation are our findings on ACTH, a measure that provides additional information about pituitary control mechanisms for cortisol release. ACTH levels trended toward overall elevation in HX+MDD smokers and appear to show almost no suppression in the abstinence condition in this group, though the interaction effect failed to reach significance. It should be noted that interpretation of our findings is complicated by the fact that HX+MDD smokers included women with both single- and multiple episodes - which contribute variability in HPA axis response-and with recency ranging from zero to 8 years. Differences in age and BMI, though not significant, may also have contributed variability in our results. We conclude that research in a larger and/or more severely depressed sample in an inpatient setting, including men as well as women, and incorporating additional measures of HPA function and frequent sampling of cortisol and ACTH over a longer period of time, ought to be conducted before the possibility of exacerbation of HPA dysregulation during smoking abstinence is dismissed. We hope that the present report will stimulate further inquiry along these lines.

\section{ACKNOWLEDGEMENTS}

This work was supported by National Cancer Institute Grant CA42730 to the first author. The authors wish to thank Elizabeth Young, M.D. for helpful advice in planning this study.

\section{REFERENCES}

Akil H, Haskett RF, Young EA, et al. 1993. Multiple HPA profiles in endogenous depression: effect of age and sex on cortisol and beta-endorphin. Biol Psychiatry 33: 73-85.

American Psychiatric Association. 1994. Diagnostic and Statistical Manual of Mental Disorders (DSM-IV), 4th edn. American Psychiatric Association: Washington, DC.

Anda RF, Williamson DF, Escobedo LG, Mast EE, Giovino GA, Remington PL. 1990. Depression and the dynamics of smoking: a national perspective. JAMA 64: 1541-1545.

Balabanis MJ, Shiffman S, Winsko LA, Gwaltney C, Waters A, Paton SM. 2001. Depression and smoking relapse. Nicotine Tob Res 3: 264-265.

Baumgartner A, Graf KJ, Kurten I. 1985. The Dexamethasone Suppression Test in depression, in schizophrenia, and during experimental stress. Biol Psychiatry 20: 675-679.

Borrelli B, Niaura R, Keuthen NJ, et al. 1996. Development of major depressive disorder during smoking-cessation treatment. J Clin Psychiatry 57: 534-538.

Carmody TP. 1989. Affect regulation, nicotine addiction, and smoking cessation. J Psychoactive Drugs 21: 331-341. 
Carroll BJ, Feinberg M, Smouse PE, Rawson SG, Greden JF. 1981. The Carroll rating scale for depression. I. Development, reliability and validation. Br J Psychiatry 138: 194-200.

Covey LS, Glassman AH, Stetner F. 1990. Depression and depressive symptoms in smoking cessation. Compr Psychiatry 31: 350-354.

Fagerström KO, Heatherton TF, Kozlowski LT. 1991. Nicotine addiction and its assessment. Ear Nose Throat J 69: 763-768.

First MB, Spitzer RL, Gibbon M, Williams JBW. 1994. Structured Clinical Interview for AXIS I DSM-IV Disorders. Biometric Research Department, New York State Psychiatric Institute: New York.

Frederick SL, Reus VI, Ginsberg D, Hall SM, Munoz RF, Ellman G. 1998. Cortisol and response to dexamethasone as predictors of withdrawal distress and abstinence success in smokers. Biol Psychiatry 43: 525-530.

Ginsberg D, Hall SM, Reus VI, Muñoz RF. 1995. Mood and depression diagnosis in smoking cessation. Exp Clin Psychopharmacol 3: 389-395.

Glassman AH. 1993. Cigarette smoking: implications for psychiatric illness. Am J Psychiatry 150: 546-553.

Glassman AH, Covey LS, Stetner F, Rivelli S. 2001. Smoking cessation and the course of major depression: a follow-up study. Lancet 357: 1929-1932.

Glassman AH, Helzer JE, Covey LS, et al. 1990. Smoking, smoking cessation, and major depression. JAMA 264: 1546-1549.

Glassman AH, Stetner F, Walsh BT, et al. 1988. Heavy smokers, smoking cessation, and clonidine. Results of a double-blind, randomized trial. JAMA 259: 2863-2866.

Hall SM, Muñoz RF, Reus VI. 1991. Smoking cessation, depression and dysphoria. NIDA Res Monogr 105: 312-313.

Hall SM, Muñoz RF, Reus VI. 1994. Cognitive-behavioral intervention increases abstinence rates for depressive-history smokers. $J$ Consult Clin Psychol 62: 141-146.

Hariharan M, VanNoord T, Greden JF. 1988. A high-performance liquid-chromatographic method for routine simultaneous determination of nicotine and cotinine in plasma. Clin Chem 34: 724-729.

Heatherton TF, Kozlowski LT, Frecker RC, Fagerström KO. 1991. The Fagerström test for nicotine dependence: a revision of the Fagerström tolerance questionnaire. Br J Addict 86: 1119-1127.

Hitsman N, Borrelli B, McChargue DE, Spring B, Niaura R. 2001. A meta-analysis of the effect of history of major depression on smoking cessation. Nicotine Tob Res 3: 271.

Hughes JR, Arana G, Amori G, Stewart F, Workman R. 1988. Effect of tobacco withdrawal on the dexamethasone suppression test. Biol Psychiatry 23: 96-98.

Janowsky DS, Risch SC. 1987. Role of acetycholine mechanism in the affective disorders. In Psychopharmacology (Berl), The Third Generation of Progress, Meltzer HY, Coyle JT, Kopin IJ, et al. (eds). Raven Press: New York; 527-533.

Johnson J, Weissman MM, Klerman GL. 1992. Service utilization and social morbidity associated with depressive symptoms is in the community. JAMA 267: 1478-1483.

Kendler KS, Neale MC, MacLean CL, Heath AC, Eaves LJ, Kessler RC. 1993. Smoking and major depression: a causal analysis. Arch Gen Psychiatry 50: 36-43.

Kinnunen T, Doherty K, Militello FS, Garvey AJ. 1996. Depression and smoking cessation: characteristics of depressed smokers and effects of nicotine replacement. J Consult Clin Psychol 64: 791-798.

Kirshbaum C, Kudielka BM, Gaab J, Schommer NC, Hellhammer DH. 1999. Impact of gender, menstrual cycle phase, and oral contraceptives on the activity of the hypothalamus-pituitary-adrenal axis. Psychosom Med 61: 154-162.
McNair DM, Lorr M, Droppleman LF. 1971. Manual for the Profile of Mood States. Educational and Industrial Testing Service: San Diego.

Niaura R, Britt DM, Borrelli B, Shadel WG, Abrams DB, Goldstein MG. 1999. History and symptoms of depression among smokers during a self-initiated quit attempt. Nicotine Tob Res 1: $251-257$.

Perez-Stable EJ, Marin G, Marin BV, Katz MH. 1990. Depressive symptoms and cigarette smoking among Latinos in San Francisco. Am J Public Health 80: 1500-1502.

Piasecki TM, Niaura R, Shadel WG, et al. 2000. Smoking withdrawal dynamics in unaided quitters. J Abnorm Psychol 109: 74-86.

Pomerleau CS, Carton SM, Lutzke ML, Flessland KA, Pomerleau OF. 1994. Reliability of the Fagerstrom tolerance questionnaire and the Fagerstrom test for nicotine dependence. Addict Behav 19: $33-39$.

Pomerleau CS, Marks JL, Pomerleau OF. 2000. Who gets what symptom? Effects of psychiatric cofactors and nicotine dependence on patterns of nicotine withdrawal symptomatology. Nicotine Tob Res 2: 275-280.

Pomerleau CS, Namenek Brouwer RJ, Pomerleau OF. 2001. Emergence of depression during early abstinence in depressed and non-depressed women smokers. J Addict Dis 20: $73-80$.

Pomerleau OF, Fertig J, Shanahan SO. 1983. Nicotine dependence in cigarette smoking: an empirically-based, multivariate model. Pharmacol Biochem Behav 19: 291-299.

Pomerleau OF, Pomerleau CS. 1984. Neuroregulators and the reinforcement of smoking: towards a biobehavioral explanation. Neurosci Biobehav Rev 8: 503-513.

Pomerleau OF, Rosecrans J. 1989. Neuroregulatory effects of nicotine. Psychoneuroendocrinology 14: 407-423.

Radloff LS. 1977. The CES-D scale: a self-report depression scale for research in the general population. Appl Psychol Measur 1: 385-401.

Ribeiro SCM, Tandon R, Grunhaus L, Greden JF. 1993. The DST as a predictor of outcome in depression: a meta-analysis. Am J Psychiatry 150: 1618-1629.

Schechter D, Bachmann GA, Vaitukaitis J, Phillips D, Saperstrom D. 1989. Perimenstrual symptoms: time course of symptom intensity in relation to endocrinologically defined segments of the menstrual cycle. Psychosom Med 51: 173-194.

Siever LJ. 1987. Role of noradrenergic mechanisms in the etiology of the affective disorders. In Psychopharmacology (Berl), The Third Generation of Progress, Meltzer HY, Coyle JT, Kopin IJ, et al. (eds). Raven Press: New York; 493-504.

Tsoh JY, Humfleet GL, Munoz RF, Reus VI, Hartz DT, Hall SM. 2000. Development of major depression after treatment for smoking cessation. Am J Psychiatry 157: 368-374.

Velten E. 1968. A laboratory task for induction of mood states. Behav Res Ther 6: 473-482.

Ward MM, Swan GE, Jack LM. 2001. Self-reported abstinence effects in the first month after smoking cessation. Addict Behav 26: $311-327$.

Weissman MM, Bruce ML, Leaf PJ, Florio LP, Holzer C. 1991. Affective disorders. In Psychiatric Disorders in America, The Epidemiologic Catchment Area Study, Robins LN, Regier DA (eds). Free Press: New York; 53-80.

Young EA, Haskett RF, Murphy-Weinberg V, Watson SJ, Akil H. 1991. Loss of glucocorticoid fast feedback in depression. Arch Gen Psychiatry 48: 693-699. 